\title{
Classification of Decision-Making Information Models as a Part of Aircraft Control System
}

\author{
O. Zaritskyi \\ Information diagnostic system institute \\ National Aviation University, \\ Kyiv, Ukraine \\ olegzaritskyi@gmail.com
}

\author{
P. Pavlenko \\ Information diagnostic system institute \\ National Aviation University, \\ Kyiv, Ukraine \\ petrpav@ukr.net
}

\begin{abstract}
Theoretical basis of decision-making models analysis information system as a part of aircraft flight control system from knowledge based system approach has been presented. As well classification of decision-making models is been described for the further development of artificial intellect "consulting" systems.
\end{abstract}

Keywords-decision making system; flight control system; artifitial intellect; profesional activity analysis

\section{INTRODUCTION}

The rapid development of cybernetics and computer science, the artificial intelligence has given significant increasing of new methods and approaches in optimization of control systems. The junction of modern control theory and artificial intelligence (AI) led to the emergence of a new direction, which is called intellectual control (management) [1]. Intellectual control includes of cause expert systems based on knowledge, in particular the systems based on the rules.

Typically, decision-making information systems had been developed in several areas, the main of which is the direction of expert systems. Currently, modern «consulting» information systems widely used $[2,3]$. It should been noted that the need for processing large amounts of data in the decision-making process, especially in the face of uncertainty, led to the emergence of a class of information systems within the methods and systems of artificial intelligence.

Onboard expert systems have classified as advisory information systems that operate on the data about the current state of the control object, as well as simulate possible scenarios of changes in the external environment. Onboard expert systems should provide a logical conclusion to the current situation and development of hypotheses that could been used for classification of decision-making models of the aircraft's crew.

\section{PROBLEM STATEMENT}

Analysis of main information system's principles of action [3] - [5], as well as implementation of control systems in parts of algorithmically, hardware and software allows us to conclude about main task of the system - synthesis of system's purposes or target solution.

Synthesis of the target solution is possible with the methods and means of obtaining information about the environment and methods for determining your own states as the control object and the control system itself.

When forming the target solution the problem of sufficiency of knowledge base, the applicability of the existing rules, possibility of their implementation, both on substance and on a constructive level has appeared. No less important is rate of incoming data and timely development of solutions templates, not only familiar with the situation, and if necessary, able to produce new knowledge.

Expert system is considered as a direction of declaration programming because information processing carried out on the level of rules instead of the level of algorithms, using programming languages like CLIPS, OPS5 etc [6].

Expert systems operate under two main constraints.

1) Continuously increasing the data rate about the state of the environment, different information systems, control system and the expert system.

2) System performance, built using a declarative rulebased programming is quite limited.

There are two ways to overcome the limitations: constantly increase data processing rate in information systems or to optimize processing algorithms. Optimization algorithms suggest identification of decision-making models and the use of artificial intellect approaches only in irrational model patterns. Therefore, the objective of the article is presenting approach for identification of decision-making models types.

There are some common approaches for classification of research objects. In the research the division of the total method or hierarchical clustering is been used. The method suggests dividing a set of objects that have some similarities, and at the same time differences. Similarity could been reflected in the criteria, the difference between them - in the accessory groups.

The considered criteria are decision-making model's attributes $C_{i}$ :

$C_{1}$ are means of operation;

$C_{2}$ are methods of operation;

$C_{3}$ is the level of control during the operation; 
$C_{4}$ is the level of decisions reasoning;

$C_{5}$ is the type of operation performed.

It's supposed to divide types of decision-making models into three groups, according to the level of certainty of the decision [8], determined by the criteria $C_{i}$ :

- $\quad$ Classical (rational) model $\left(M_{1}\right)$;

- $\quad$ Behavioral model $\left(M_{2}\right)$;

- $\quad$ Irrational model $\left(M_{3}\right)$.

Thereby decision-making models could been described as a function of five variable $C_{i}$ by common equation

$$
M_{j}=f\left(C_{i}\right)
$$

The main objective of research is to clarify possible combinations of criteria's value, relationship between the criteria and using the classification methods group possible variety of decision-making models.

\section{Problem Classification Solution of Decision- MAKING INFORMATION MODELS}

Described in [7] the functional model of the essence "model solution" combines approaches of scientists M. Woodcock and Francis D. on classification levels of decisionmaking and F. Findler on the degree of structuring of the problem and, as a consequence necessarily level of creativity required for its solution.

Level of decision by Francis D. roughly classified into ordinal scale from routine, which involves a decision in complete certainty from input and output parameters point of view to innovation, which characterized by almost complete uncertainty inherent to the development of new technologies and methodologies.

Tools and methods for performing operations been measured in ordinal scale from determined to undetermined, which is necessary for solution of innovative problems that were not been solved before, or existing methods and tools should be improved or developed.

The level of control been assessed from the constant, direct control during the operation to complete lack of control, when creative and innovative problem are been solved.

Possible combinations of attributes' estimates of the essence - "model solution" presented in Table I and used as input in the classification analysis. Rating scales described in detail in the article [8].

TABLE I. COMBINATIONS OF ESSENCE "MODEL SOLUTION" ATTRIBUTE ESTIMATES

\begin{tabular}{|c|c|c|c|c|}
\hline $\boldsymbol{C}_{\mathbf{1}}$ & $\boldsymbol{C}_{\mathbf{2}}$ & $\boldsymbol{C}_{\mathbf{3}}$ & $\boldsymbol{C}_{\mathbf{4}}$ & $\boldsymbol{C}_{\mathbf{5}}$ \\
\hline 1 & 1 & 1 & 1 & 1 \\
\hline 1 & 1 & 2 & 2 & 2 \\
\hline 1 & 1 & 2 & 2 & 3 \\
\hline 1 & 1 & 1 & 1 & 3 \\
\hline 1 & 1 & 2 & 1 & 3 \\
\hline 1 & 1 & 1 & 2 & 3 \\
\hline 1 & 1 & 1 & 1 & 2 \\
\hline
\end{tabular}

\begin{tabular}{|l|l|l|l|l|}
\hline 1 & 1 & 1 & 2 & 2 \\
\hline 1 & 1 & 1 & 2 & 1 \\
\hline 1 & 1 & 2 & 1 & 1 \\
\hline 1 & 1 & 2 & 2 & 1 \\
\hline 1 & 1 & 2 & 1 & 2 \\
\hline 2 & 2 & 3 & 3 & 4 \\
\hline 2 & 2 & 4 & 3 & 4 \\
\hline 2 & 2 & 3 & 3 & 5 \\
\hline 2 & 2 & 4 & 3 & 5 \\
\hline 2 & 2 & 2 & 3 & 4 \\
\hline 2 & 2 & 2 & 3 & 5 \\
\hline 3 & 3 & 4 & 4 & 6 \\
\hline 3 & 3 & 4 & 4 & 7 \\
\hline 3 & 3 & 5 & 4 & 6 \\
\hline 3 & 3 & 5 & 4 & 7 \\
\hline 2 & 2 & 4 & 4 & 6 \\
\hline 2 & 2 & 4 & 4 & 7 \\
\hline 2 & 2 & 5 & 4 & 6 \\
\hline 2 & 2 & 5 & 4 & 7 \\
\hline 2 & 3 & 4 & 4 & 6 \\
\hline 2 & 3 & 4 & 4 & 7 \\
\hline 3 & 2 & 5 & 4 & 6 \\
\hline 3 & 2 & 5 & 4 & 7 \\
\hline
\end{tabular}

Since the attributes evaluation of the essence are been measured by categorical values, as a function of distance in agglomerative clustering procedure were used inconsistencies percent and weighted average pairwise rules for hierarchical clustering.

Result of clustering shown in Fig. 1. Numbers of attributes estimates combinations of the essence "model solution" marked $C_{i}\{1 \ldots 30\}$ corresponding to the 30 experimental combinations [8].

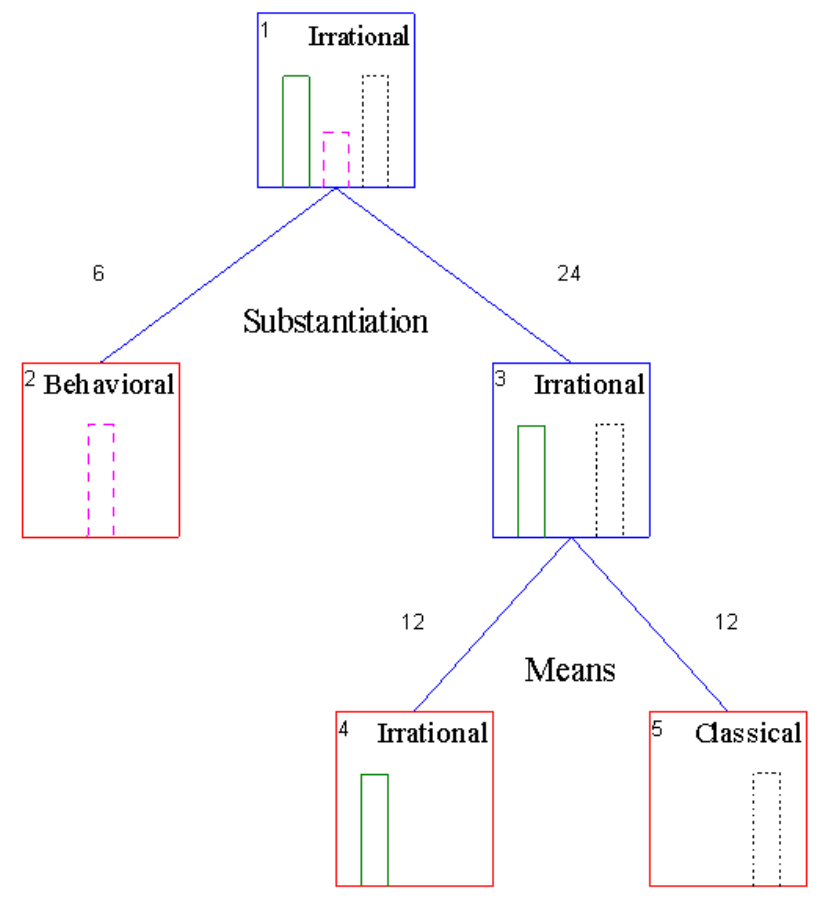

Fig. 1. Classification graph tree of decision model.

Combinations $C_{i}\{1 \ldots 12\}$ correspond to the classical decision-making model, as characterized by certainty of methods and means of operation. Decision usually takes place 
under maximum supervision of the head or systems and its justification is been based on standardized procedures and instructions.

Combinations $C_{i} \quad\{13 \ldots 18\}$ describe the behavioral decision-making model, which is characterized by some uncertainty in terms of means and methods. The person, who makes the decision, is almost within the nominal control, and the type of operation is between the process and consulting activities. It might be applied partly for researches.

Combinations of estimates $C_{i}\{19 \ldots 30\}$ describe irrational decision-making model, which is characterized by considerable uncertainty both in terms of methods and tools and in the implementation of the researches: applied, original, providing innovative substantiation solutions level.

As a result of the clustering attribute's set were divided into three subsets that correspond to the three defined type of decision models.

Scattering diagram (Fig. 2) of canonical values for pairs of discriminant function values provides a graphical representation of the distribution (group) of models. Combinations of estimates that are the same model types are localized in certain areas of the plane.

The distance between centroids of irrational and behavioral decision-making model less than the between classical and behavioral models, indicating a weak boundary between the first two models of decision.

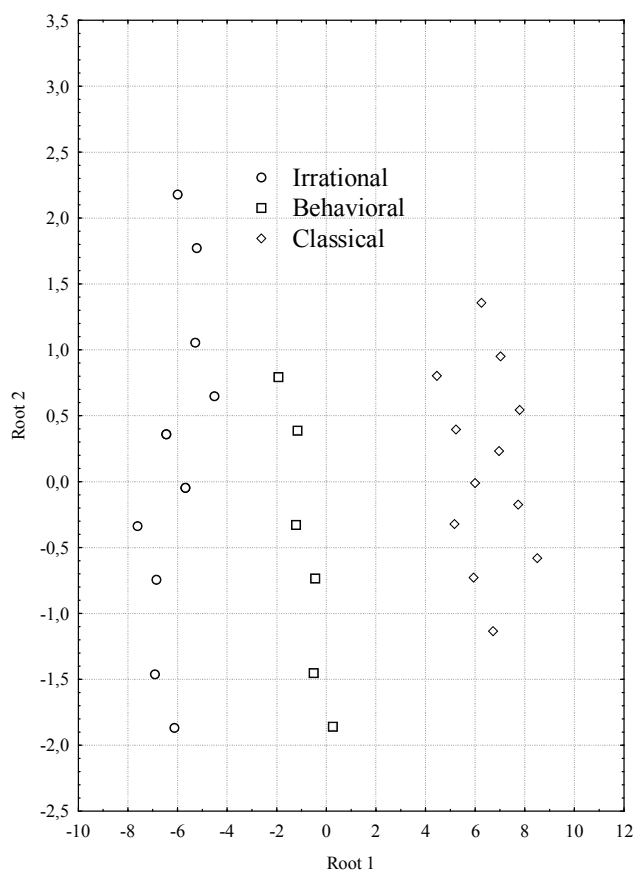

Fig. 2. Scattering diagram.

The results of classification trees (Fig. 1) as a method of discriminant dimensional clustering for categorical predictors using the CART method correlate with the cluster analysis results (Fig. 2) in part of clear separation of possible solutions into three groups according to the attribute values.

Conceptual expert system structural model (Fig. 3) was presented in the article [9]. User's interface allows to organize data input or their loading from database into module, responsible for data calculating, and dialog with users. Method of decision interpreting allows getting reasons of the decision by the system. Working memory keeps facts, which are been creating during working of entity calculation algorithm [10]. Inference engine - program component of the system, responsible for inference, operating with rules and facts. It ranks rules and fulfill rule with the highest priority.

Working list of rules contains actual rules in order of priority, if their patterns satisfy facts or objects from working memory.

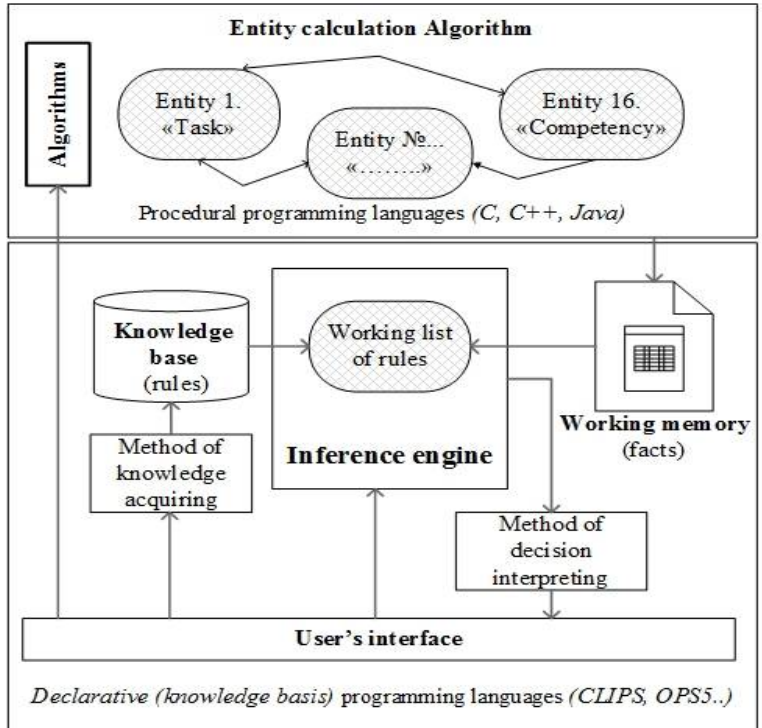

Fig. 3. Expert system structure, basis on the rules.

Authors presented the results of formalizing information decision-making models by introduction and description of the entities and the attributes of the models using linguistic variables of the fuzzy sets theory in order to further use in flight control systems or other information system [10]. We'll present the criteria in terms of the linguistic variables using CLIPS language:

$\mathrm{C}_{1}, \mathrm{C}_{2}$ - means (methods) of operation:

(deftemplate Execution_methods (resources)

010 points

((detr (z 1 4))

$(\operatorname{detp}($ pi 3 5))

; determine completely

(udet (s 6 10))))

; determine partly

; undetermined

$\mathrm{C}_{3}$ - level of control during the operation.

(deftemplate Control_level

010 points

((ccon (z 14$))$

(absc (s 6 10))

; constant control

(mang not [ ccon or absc ]))) ; management

$\mathrm{C}_{4}$ - level of decisions reasoning

(deftemplate Reasoning_level

010 points

((rout $(\mathrm{z} 14)) \quad$; routine

(sele (pi 3 5)) ; selective 


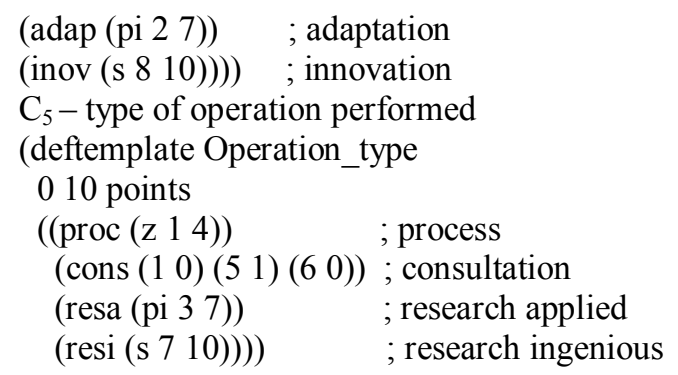

Decision-making models in terms of linguistic variables using CLIPS language are presented in Fig. 4.

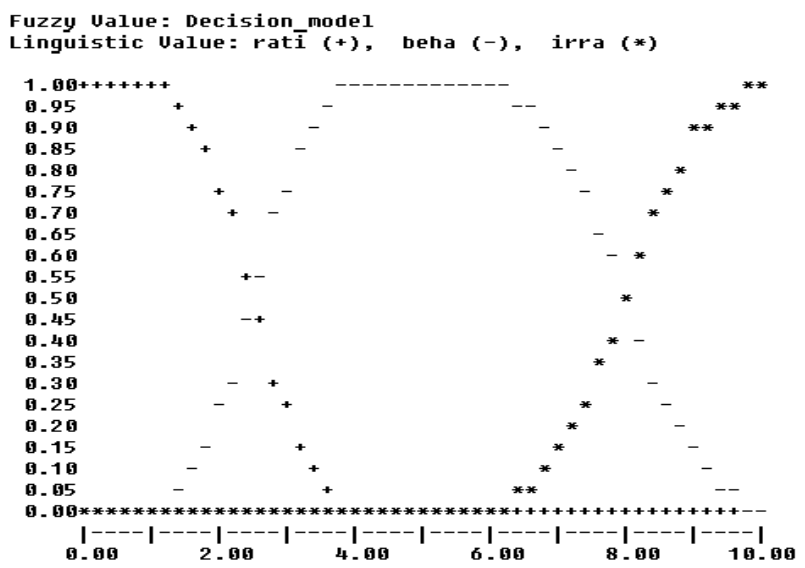

Fig. 4. Graphical presentation of linguistic variables "Decision-making models".

The expert system provides activation of appropriate knowledge base for the definition decision-making models and calculation their numerical assessments using rules.

The rules were developed for the expert system are based on FUZZYClips annotation:

defrule Rational_model

(Execution methods detr)

(Execution resources detr)

(Grounding_level rout or sele)

(Control_level ccon), (Operation_type proc)

$=>($ assert $($ Decision_model rati $)))$

(defrule Irrational model

(Execution_methods detp or udet)

(Execution_resources detp or udet)

(Grounding level adap or inov)

(Control_level absc), (Operation_type resi)

$=>\quad($ assert $($ Decision_model irra $)))$

(defrule Behavioral model

(Execution methods detp)

(Execution_resources detp)

(Grounding level sele or adap)

(Control level mang)

(Operation_type cons or resa)

$=>($ assert $($ Decision_model beha $)))$
The result of decision-making models classification using developed expert system and algorithms (rules) are presented on (Fig. 5). As well, on Fig. 5 initial states of the creteria's values are presented.

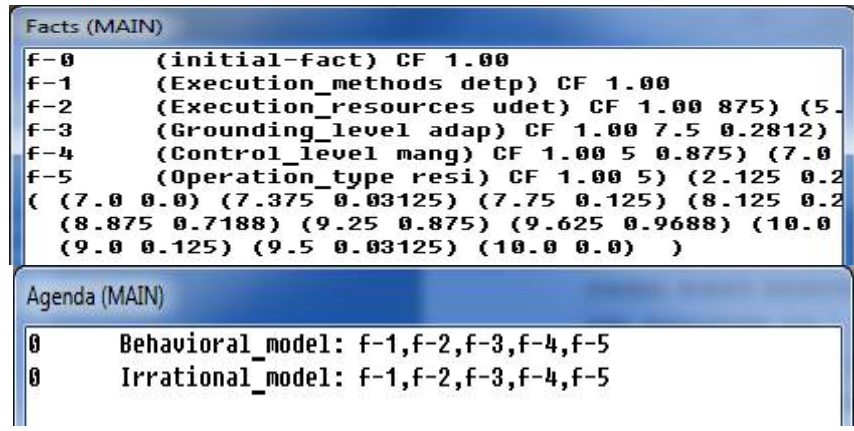

Fig. 5. Result of classification.

\section{CONCLUSION}

The analysis of possible combinations of attributes' assessments of the essence - "model solution" and their further classification allowed to describe three types of decision-making models: classic, behavioral and irrational. On the bases of the presented criteria and types of models expert system rules were developed.

\section{REFERENCES}

[1] S.N. Vasyl'ev, A.K. Zherlov, E.A. Fedosov, and B.E. Fedunov, Intelligent control of dynamic systems. Moscow, Fyzmatlyt, 2000, 420 p. (in Russian)

[2] Y.M. Makarova and V.M. Lokhyna, Intelligent automatic control system. Moscow, Fyzmatlyt, 2001, 375 p. (in Russian)

[3] E.A. Fedosov, Onboard expert systems of 5th generation tactical aircrafts. Moscow, Nauchno-ynformatsyonnyj tsentr HosNYYAS [Scientific Information Center], 2002, 265 p. (in Russian)

[4] Fang Ke, A. Proletarsky, and K. Neusipin, "Selection of Measured Signals in the Navigation Measuring Complex." Journal of Measurement Science and Instrumentation. December 2011, vol. 02, no. 04 . pp. 346-348.

[5] A. Proletarsky, and K. "Neusipin Reserch scalar filtering algorithm with selforganization method for modeling control system." Science \& military. 2010, vol. 5, no. 2, pp. 17-21

[6] CLIPS, Reference Manual. vol. II. Advance programming guide. 2007, $293 \mathrm{p}$.

[7] O.V. Zarits'kyj, "Informational modeling of decision making process." Software engineering, Kyiv, NAU, 2015, no. 1(25), pp. 56-61. (in Ukrainian).

[8] O.V. Zarits'kyj, "Theoretical basis of decision-making model formalizing in frame of professional activity's estimation algorithm with help of information technology." Informatization and control questions, Kyiv, NAU, 2015, no. 3(51), pp. 51-55. (in Ukrainian).

[9] O.V. Zarits'kyj. "Theoretical basis of professional activity analysis and estimation expert systems development." Electronics and control system, Kyiv, NAU, 2015, no. 2(44), pp. 103-106. (in Ukrainian).

[10] O.V. Zarits'kyj. "The formalization of decision-making models in expert information system of professional activity assessment." Chasopys «Visnyk inzhenernoi akademii Ukrainy». [Journal of Engineering Academy of Ukraine]. Kyiv, NAU, 2016, no. 1, pp. 66-71. (in Ukrainian). 